\section{COMBO Dual Therapy Stent: a novel technology in interventional cardiology}

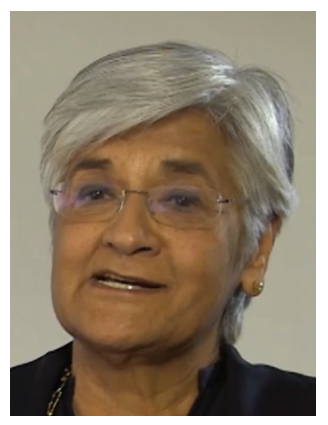

Renu Virmani* speaks to Ellen Clarke, Commissioning Editor: R Virmani is a world-renowned cardiovascular pathologist and is recognized as a leading researcher in the field of cardiovascular disease treatments. Presented with the 2012 TCT Career Achievement Award by the Cardiovascular Research Foundation, as well as honorary awards by ESC and EuroPCR for her dedicated research work, she proctors across the globe delivering more than 800 presentations at scientific meetings and has authored or coauthored over 600 publications in peer-reviewed journals and is also a manuscript reviewer for many major scientific journals.

First draft submitted: 16 June 2015; Accepted for publication: 7 October 2015;

Published online: 21 December 2015

Q Please share with us a bit about your career \& how you became involved in the field of interventional cardiology

It all began when I first came to the USA in 1974, when I met cardiac pathologist, Dr William C Roberts, who then became my mentor and inspired me. Even before entering into my residency, I already wrote two or three papers with him in the first 8 months. Bill later helped me enroll in the residence program at the George Washington University and 2 years into the program, he offered me a fellowship at the NIH where he worked as the Chief of Cardiovascular Pathology, NHLBI (National Heart, Lung, and Blood Institute), NIH (National Institutes of Health, Bethesda, MD, USA). So that is really where I began my career as a cardiac pathologist and learned about interventional cardiology, the latter is an interesting story. Marty Leon was a fellow in the clinical division of the NHLBI, NIH at the time of my fellowship, so really we were both fellows at the same time. After completion of my fellowship, I moved and joined the Armed Forces Institute of Pathology while Marty went to Yale, but he later returned to the NIH. Unexpectedly one day, I received a call from Marty about some Palmaz-Schatz stent (which I knew nothing about) and he asked me if I could cut and show the histologic changes that may have occurred around the stent, after which he had explained its usefulness and operation. It was certainly a challenge for me but that was also the defining moment of how I became involved in interventional cardiology.

\section{Q What are the key features of the COMBO Dual Therapy Stent?}

First of all, it delivers a sirolimus drug that controls restenosis and the drug is eluted via an abluminal biodegradable polymer, which degrades in 90 days and also reduces inflammatory responses as compared with durable polymers.

Second, the COMBO stent is coated by CD34 antibody, which is really unique because it attracts endothelial progenitor cells that are in the circulation, facilitating surface endothelialization at a rapid speed. Prior to the availability of COMBO, the

\section{KEYWORDS}

- biodegradable polymer

- CD34 antibody

- endothelium

*CVPath Institute, 19 Firstfield Road, Gaithersburg, MD 20878, USA; rvirmani@cvpath.org

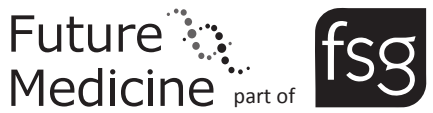


endothelial progenitor cell (EPC) capture concept (without drug coating) was studied under the Genous Stent and it was very exciting to see that not only did the antibody attract endothelial cells, it also helped reduce thrombus formation in a human arteriovenous (AV) shunt model.

\section{Q How does endothelial progenitor cell- capturing technology promote accelerated healing of the vessel wall?}

Often when we talk about vessel healing, we say there should be smooth muscle cell formation and less fibrin, but the most important element of healing really is surface endothelialization. The EPC-capturing technology by the CD34 antibody facilitates endothelialization, which perhaps also attracts adjacent endothelial cells on the vessel wall. Of course, the presence of fibrin beneath the endothelium prevents restenosis, but its presence is of no consequence as the luminal surface is covered by the endothelium. This endothelium, which has been shown by us to be functional because it produces antithrombotic agents, like endothelial nitric oxide synthase (eNOS), as well as forms tight junctions (PECAM1 expression), which are likely facilitated by the capture of the circulating EPCs as compared with having only the endothelial cells available from the vessel wall, which will likely take longer time as they need to proliferate (because of loss of endothelial cells during the procedure and the presence of drug) and only then be able to cover the stent surface.

\section{Q What are the advantages of the COMBO} Dual Therapy Stent over existing technologies? It all starts with endothelialization, which is what is promoting healing. In real life everyday practice, we deal with patients who really cannot tolerate long-term dual antiplatelet therapy (DAPT) because of bleeding issues. So here we have a prohealing drug eluting stent (DES) unlike other conventional DES (with only an antiproliferative effect), that is unique and provides the option of short-term DAPT to reduce bleeding risks, but also minimizes the risk of late stent thrombosis. Of course, we need more clinical data for validation but several large-scale trials are already ongoing and it would be very exciting to see those results.

\section{Q Can you highlight some of the success} seen in recent clinical trials of this EPC capture technology?

Before the availability of COMBO, the EPC capture concept was studied in humans under the Genous Stent, which proved its safety, especially in stent thrombosis (ST) elevation myocardial infarction (STEMI) patients where stent thrombosis (ST) remained low. For example, in the Genous Acute Myocardial Infarction (AMI) study, the ST rate remained at a stable and low $1.3 \%$ from the first year out to 3 years without any additional thrombotic events. Also, from the Evaluation of healinG by Optical coherence tomography (EGO) Genous study, 70\% strut coverage was shown as early as 14 days, which could explain why ST rates are low, because the stent is sufficiently covered.

With COMBO, we have not seen any ST events reported up to 4 years from the REMEDEE and EGO COMBO trials, and in terms of clinicallydriven target lesion revascularization, the two trials showed 7.3 and $0 \%$, respectively, which look excellent and promising. Chances are, this stent may also prevent neoatherosclerosis but we do not know at this moment and further validation is needed.

Looking back at the preclinical findings in animal models at 28 days comparing COMBO with Xience, endothelial coverage was significantly better with COMBO as compared with Xience, while conversely, the overall uncovered struts were much lower in $\mathrm{COMBO}$ versus Xience. In humans, EGO COMBO also demonstrated a healthy prohealing profile with near complete coverage by 9 months. What is very exciting about these studies is that neointimal regression is observed from 9 to 24 months, a result that is unseen with any other metallic DES currently on the market.

\section{Q Are there any ongoing clinical trials you can} tell us about?

Including REMEDEE and EGO COMBO, which were discussed earlier, there are also several ongoing large-scale trials totaling $7000+$ patients to demonstrate the clinical benefits of the COMBO stent. For example, we have the HARMONEE randomized control trial involving nearly 600 patients from Japan and the USA in a harmonized fashion for simultaneous approval in both countries, the REMEDEE European Registry involving 1000 patients predominantly in acute coronary syndrome (ACS), as well as the MASCOT global registry involving 2500 patients to look at how the COMBO performs in patients over a diverse spectrum of background.

I am particularly excited about two specific trials, namely REDUCE and COSTA. 
REDUCE is, in my opinion, a pioneer trial as it focuses strictly on ACS patients and assesses the feasibility of lowering DAPT regimen. As we know, ACS patients are the ones who get into more trouble with long-term DAPT, but at the same time require long-term DAPT by the nature of the disease, so it is exciting to have a trial that looks at a 3-month DAPT duration in the toughest setting. It would be good to look at the results and say 'only $3 \mathrm{~m}$ DAPT and still prevent late stent thrombosis!'

COSTA, in the meantime, is a trial that looks at patients with atrial fibrillation who are indicated for chronic warfarin medication but are also susceptible to increased and severe bleeding risks. The study is designed to assess the feasibility of reducing DAPT regimen from on label device DAPT recommendation down to 3 months to potentially help reduce bleeding risk.

So, all in all, it is very encouraging to see that the COMBO clinical program is continually expanding to study and explore the potential clinical benefits over a broad variety of patients.

\section{Q What effects will this new technology have on the future of interventional cardiology?}

I think this technology is unique and there is no other stent on the market that allows active healing by attracting EPCs, so I think this is very exciting and this is a new phase of DES technology - abluminal bioabsorbable polymer + CD34 antibody-attracting endothelial progenitor cells - and I think patients are likely to benefit from it.

Q What advancement do you see in this field over the next 5 years?

I think we will see a reduction in or even a complete absence of polymer usage in DES. I think the other exciting technology in a way is drugeluting balloon, which is already here, but I think that applying balloons with sirolimus would be an advancement as all current drug-eluting balloon are coated with paclitaxel. Then, of course, there are the bioabsorbable scaffolds, which I think at the moment are not ideal as they are quite thick at $150 \mu \mathrm{m}$. We already see the thinner next-generation bioabsorbable scaffolds reduced to 120 or $100 \mu \mathrm{m}$, and the danger is that they will show more fractures, only time will tell.

\section{Disclaimer}

The opinions expressed in this interview are those of the interviewee and do not necessarily reflect the views of Future Medicine Ltd.

\section{Financial \& competing interests disclosure}

The author has no relevant affliations or financial involvement with any organization or entity with a financial interest in or financial conflict with the subject matter or materials discussed in the manuscript. This includes employment, consultancies, honoraria, stock ownership or options, expert testimony, grants or patents received or pending, or royalties.

No writing assistance was utilized in the production of this manuscript. 